Review

\title{
Calcineurin Orchestrates Hyphal Growth, Septation, Drug Resistance and Pathogenesis of Aspergillus fumigatus: Where Do We Go from Here?
}

\section{Praveen R Juvvadi ${ }^{1, *}$ and William J Steinbach ${ }^{1,2}$}

1 Department of Pediatrics, Division of Pediatric Infectious Diseases, Duke University Medical Center, Durham, NC 27710, USA; E-Mail: bill.steinbach@duke.edu

2 Department of Molecular Genetics and Microbiology, Duke University Medical Center, Durham, NC 27710, USA

* Author to whom correspondence should be addressed; E-Mail: praveen.juvvadi@duke.edu; Tel.: +919-681-1504; Fax: +919-668-4859.

Academic Editor: Ying-Lien Chen

Received: 23 November 2015 / Accepted: 11 December 2015 / Published: 16 December 2015

\begin{abstract}
Studies on fungal pathogens belonging to the ascomycota phylum are critical given the ubiquity and frequency with which these fungi cause infections in humans. Among these species, Aspergillus fumigatus causes invasive aspergillosis, a leading cause of death in immunocompromised patients. Fundamental to A. fumigatus pathogenesis is hyphal growth. However, the precise mechanisms underlying hyphal growth and virulence are poorly understood. Over the past 10 years, our research towards the identification of molecular targets responsible for hyphal growth, drug resistance and virulence led to the elucidation of calcineurin as a key signaling molecule governing these processes. In this review, we summarize our salient findings on the significance of calcineurin for hyphal growth and septation in A. fumigatus and propose future perspectives on exploiting this pathway for designing new fungal-specific therapeutics.
\end{abstract}

Keywords: Aspergillus fumigatus; calcineurin; hyphal growth; septation; virulence; FK506; cyclosporine A; phosphorylation; drug target; drug resistance 


\section{Introduction}

Invasive fungal infections are one of the most common causes of death in immunocompromised patients [1]. Calcineurin is a target of immunosuppressive agents, tacrolimus (FK506) and cyclosporine A (CsA), mediated via their respective immunophilins, FKBP12 and cyclophilin A [2,3]. Genetic studies in fungi have established the molecular mechanism of calcineurin inhibition by the CsA-cyclophilin A and FK506-FKBP12 complexes [2,4,5]. However, these currently available anti-calcineurin agents are not ideal for treating invasive fungal infections due to their host calcineurin cross-reactivity and subsequent immunosuppressive effects [3]. Therefore, an in depth understanding of calcineurin and its functions in fungi is essential to exploit the calcineurin pathway to formulate novel fungal-specific targeting approaches.

Calcineurin is a heterodimer comprised of a catalytic $(\mathrm{CnaA})$ and regulatory $(\mathrm{CnaB})$ subunit [6]. It is also known as protein phosphatase $2 \mathrm{~B}$ and is activated through the binding of $\mathrm{Ca}^{2+}$-calmodulin $(\mathrm{CaM})$ [7]. Among the known serine/threonine protein phosphatases, calcineurin is the only phosphatase that requires $\mathrm{Ca}^{2+}$ and $\mathrm{CaM}$ for its enzymatic activity and exhibits restricted substrate specificity [7-9]. Though highly conserved from the lower eukaryotes to humans, calcineurin performs diverse functions in different organisms [10]. For instance, in the yeasts Saccharomyces cerevisiae and Schizosaccharomyces pombe, it regulates adaptation to a variety of environmental stresses, cation homeostasis, morphogenesis, cell wall integrity, and mating [11-19]. In the pathogenic yeasts Cryptococcus neoformans and Candida albicans, calcineurin regulates growth at alkaline $\mathrm{pH}$, elevated temperature, membrane stress, dimorphism, mating and virulence [20-24]. In filamentous fungi, it regulates hyphal growth, stress adaptation, sclerotia and appresoria development and cell wall integrity [25-34].

As a drug target, calcineurin's roles have been extensively investigated in human pathogens such as $C$. neoformans, C. albicans and other Candida species, A. fumigatus, and Mucor circinelliodes [35-39]. Understanding the calcineurin signaling network in the various fungal pathogens is beneficial for designing new targeted therapeutics. Filamentous fungi have a single set of genes encoding the calcineurin catalytic and regulatory subunits and are therefore ideal model systems to study the role of these subunits in vivo. Critically investigating the calcineurin pathway in A. fumigatus to identify residues indispensable for calcineurin activity in vivo will pave the way for devising new drug targets for combating invasive aspergillosis. In this review, we summarize our latest findings on calcineurin functions in this human pathogen and how these relate to its cellular localization and influence on the various downstream events and targets.

\section{Calcineurin Is Essential for Hyphal Extension and Septation}

Previous studies from our laboratory and from others have shown that deletion of the catalytic subunit of A. fumigatus calcineurin (cnaA) resulted in blunted, hyperbranched and irregularly septated hyphae, indicating the requirement of calcineurin for proper hyphal extension and regular septation [40-42]. Double deletion of both the subunits (cnaA and cnaB) caused more severe defects in germination and septation [42]. To better understand how and to what extent calcineurin controls septum formation and hyphal growth, more detailed studies on visualizing the localization patterns of CnaA and 
$\mathrm{CnaB}$ in hyphae demonstrated their localization at the active points of hyphal growth - the hyphal tip and hyphal septum $[42,43]$. Calcineurin localization was also observed in small punctate structures that were motile and moved retrograde from the hyphal tip to the point of septation (Figure 1) [42]. To address calcineurin's role during septum formation, we visualized the actin contractile ring during septum formation by co-localization of fluorescently labeled $\mathrm{CnaA}$ and actin (LifeAct) [42]. Localization studies with actin also confirmed its co-localization with calcineurin early during the septation process and after the formation of mature septa. This result indicates that the calcineurin complex participates in early septation process and may be used for activation of the cell wall biosynthetic components. No variations were noted in the contractile actin ring formation in the $\Delta c n a A$ $\triangle c n a B$ double mutant strain, indicating that the septation defects may be caused during the primary or secondary wall formation. The cell wall defects observed in the calcineurin deletion strains could largely be due to inactivation of the PKC (Protein kinase C) pathway and the Crz1 transcription factor, which is activated by calcineurin.

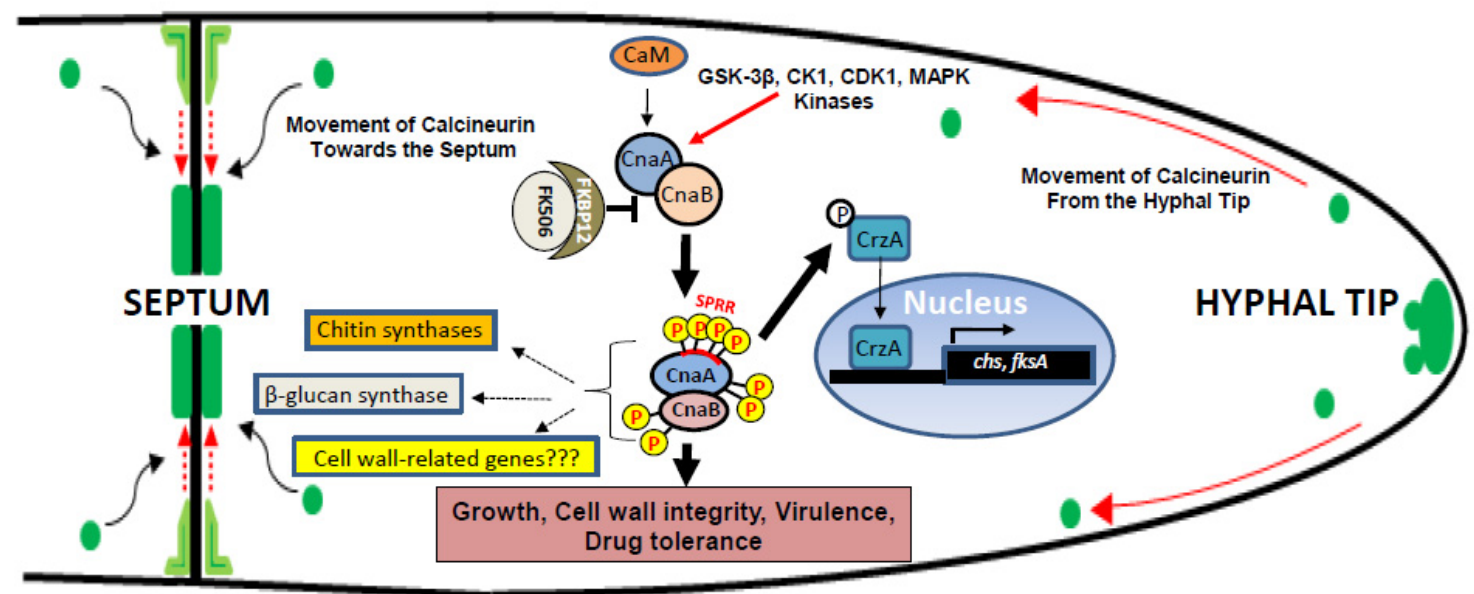

Figure 1. Schematic model of calcineurin localization and function in A. fumigatus. Calcineurin localizes to puncta which are motile and move retrograde from the hyphal tip to the point of septation and localize on either sides of the septum as a disc around the septal pore. Comprised of the catalytic ( $\mathrm{CnaA})$ and the regulatory subunit $(\mathrm{CnaB})$, calcineurin binds to calmodulin (CaM) and is activated through phosphorylation by kinases (GSK-3 $\beta$, CK1, CDK1, MAPK). Phosphorylation occurs at the Serine Proline Rich Region (SPRR) and the C-terminus. $\mathrm{CnaB}$ also undergoes phosphorylation at the N-terminus. Calcineurin is inhibited by the binding of the immunosuppressant-immunophilin complex (FK506-FKBP12). Phosphorylated calcineurin complex may dephosphorylate the transcription factor CrzA and translocate it into the nucleus to activate the transcription of cell wall biosynthesis related genes (chs genes and $f k s A$ ). Similarly, the phosphorylated calcineurin complex may also interact with cell wall proteins directly in a phosphorylation-dependent manner to regulate cell wall integrity, virulence and drug resistance.

Interestingly, the calcineurin deletion strains do form septa, but very irregularly and with shorter apical, subapical and basal hyphal compartments. While the septa in the $\triangle c n a A$ and $\triangle c n a B$ strains were 
similar, the double mutant showed a large percentage of incomplete septa or wavy septa [42]. Previous reports on the characterization of the chitin synthase double mutant ( $\triangle \operatorname{chs} A \Delta c h S C)$ and a $\Delta \operatorname{chs} B$ single mutant in Aspergillus nidulans also showed irregular septation and wavy/incomplete septa $[44,45]$ resembling the calcineurin double mutant. Recent deletion analysis of multiple genes belonging to class I (chs $A$, $\operatorname{chs} B, \operatorname{chs} C$ and $\operatorname{chs} G$ ) and class II (chsF, $\operatorname{chs} D, \operatorname{csm} A$ and $\operatorname{csm} B$ ) chitin synthase family in A. fumigatus also revealed defects in hyphal morphology, conidiation and cell wall organization [46]. Although the specific role of calcineurin during septum formation is yet unknown, similar septation and cell wall organization defects observed with the chitin synthase deletion strains in A. nidulans and A. fumigatus implicates calcineurin as playing a role in the activation of chitin synthases or chitin and $\beta$-glucan assembly at the hyphal septum (Figure 1). Supporting this notion, we also found that the calcineurin double mutant has less $\beta$-glucan at the septa during early periods of growth when compared to the single mutants [42].

\section{Septal Localization: A Key to Calcineurin Function}

Hyphae of A. fumigatus extend and invade host lung tissue in order to cause invasive aspergillosis. Septation is an important event for hyphal extension and the calcineurin complex appears to regulate this process through a yet undefined mechanism in filamentous fungi. Although the catalytic subunit (CnaA) localizes at the hyphal septum, it alone is unable to complement for hyphal growth and requires the regulatory subunit $(\mathrm{CnaB})$ for the activity and function at the hyphal septum [42]. Catalytic null mutations generated in $\mathrm{CnaA}$ do not complement the hyphal growth defect but do not affect the localization of the complex at the hyphal septum $[42,47]$. Though the complex localizes at the hyphal septum, it needs to be enzymatically active to regulate proper hyphal growth. Taken together, both localization and activity of the calcineurin complex at the hyphal septum are important for its function (Figure 1).

To understand the mechanistic basis for calcineurin localization and identify critical domains essential for its localization and function at the hyphal septum, we performed site-directed mutagenesis of conserved residues in the linker between the $\mathrm{CnaA}$ catalytic domain and $\mathrm{CnaB}$ binding helix (T359P, H361L, L365S), the PxIxIT binding motif (352NIR354-AAA), CnaB binding helix (V371D), calmodulin binding domain (CaMBD; 442RVF444-AAA) and the unique serine-proline rich region (SPRR; 406-SPSAPSPPLP-417) in the linker region between the CnaB binding helix and CaMBD of CnaA $[42,47]$. These mutations revealed that only the PxIxIT substrate binding motif residues 352NIR354 are required for septal localization, confirming that calcineurin localizes at the septum through interaction with as yet unknown substrate(s) at the septum.

To examine if the immunosuppressant and immunophilin complex is also required for localizing the CnaA-CnaB complex at the hyphal septum, we also treated it with FK506 and CsA and examined it for any change in the calcineurin localization pattern. FK506 or CsA treatment did not alter the localization of the calcineurin complex from the septa, indicating that localization of the complex at the septa is not dependent on binding to the immunophilins [42]. However, since FK506 inhibits the activity of calcineurin, it became evident that functional activity of the calcineurin complex is absolutely required at the hyphal septum in order to regulate proper hyphal growth. In support of this observation, we also recently showed that FKBP12 localized to the hyphal septum only in the presence of FK506 [48]. 
Since the calcineurin complex selectively concentrates at the hyphal septum, we are pursuing future work to identify the targets of calcineurin at the hyphal septum to clarify the exact function of this complex in regulating septation and hyphal growth. We expect these studies will lead to identification of other targets of calcineurin.

\section{Calcineurin Phosphorylation: A Unique Modification}

Various functional aspects of calcineurin have been studied in several model organisms and its functional domains described, but phosphorylation as a mechanism of calcineurin function was never before examined in a human pathogen. Our phosphoproteomic analyses revealed A. fumigatus CnaA phosphorylation in vivo at a unique cluster of serine and proline residues, that we termed the Serine Proline Rich Region (SPRR), that is specific to filamentous ascomycetes (Figure 1) [47]. Phosphorylation at the four serine residues in proximity to the proline residues may induce secondary structure conformation in the molecule, facilitating interactions with other proteins. Furthermore, mutation of the phosphorylated serine residues in the SPRR to block the phosphorylation of CnaA in vivo caused increased branching and reduction in hyphal growth [47], confirming the significance of phosphorylation for calcineurin function and activity in regulating hyphal growth.

Interestingly, phosphorylation was reduced in both the catalytic and the regulatory calcineurin subunits following treatment with FK506 [47]. It is possible that the FK506-FKBP12 complex may indirectly cause an inhibitory effect on the kinase that phosphorylates calcineurin at the SPRR and also at the N-terminus of CnaB. Based on the phosphorylation of CnaA residues in both the SPRR and the C-terminus, we speculated that more than one kinase is responsible. Proline-directed kinases such as GSK-3, CDK1 and MAP kinase were able to phosphorylate all four serine residues in the SPRR (Figure 1) [47]. While GSK-3 $\beta$ alone phosphorylated Ser413 in the SPRR, CK1 alone phosphorylated Ser406, and a combination with GSK-3 $\beta$ led to phosphorylation of other serine residues (Ser408 and Ser410), revealing that the prephosphorylation of the Ser406 residue by CK1 may trigger the subsequent phosphorylation of Ser408 and Ser410. Inhibitors for GSK-3 $\beta$ and CK1 blocked phosphorylation at Ser406 only, but not the other serine residues, leading to the notion that other yet unknown kinase may also phosphorylate the CnaA SPRR in vivo. The observed phosphorylation of CnaA SPRR by CDK1 and MAP kinase in vitro strengthens this possibility. However, further analyses are required to specifically identify the kinase/s involved in CnaA phosphorylation. In another recent study, we also showed differential phosphorylation of calcineurin following treatment with two echinocandin antifungals, caspofungin and micafungin [49]. We found that calcineurin phosphorylation at the SPRR is important for regulation of "paradoxical growth" at higher concentrations of caspofungin. This suggests that phosphorylation of calcineurin could be the mechanism underlying the phenotypic paradoxical growth. Further work is required to clearly understand how these dynamic changes in the phosphorylation status of CnaA following treatment with the two echinocandins influences calcineurin's inactivation/activation and its subsequent interaction with downstream targets.

\section{Calcineurin as an Antifungal Drug Target: Future Perspectives}

Due to limitations in the currently available antifungal armamentarium, novel therapeutic strategies are needed to combat invasive aspergillosis [50,51]. Calcineurin inhibitors are attractive as new 
antifungal agents due to their specific mode of action from other antifungal classes (polyenes, triazoles, echinocandins) that would target the top of a critical cell signaling pathway, efficacy against emerging azole- and echinocandin-resistant strains [52], and synergistic nature with existing antifungals such as the echinocandin antifungal caspofungin [53]. Moreover, the observed "paradoxical growth effect" at higher concentrations of caspofungin is abolished in the presence of FK506 and by genetic mutations of calcineurin, implicating interaction of calcineurin with cell wall biosynthetic machinery [54]. However, currently available calcineurin inhibitors bind to immunophilins, leading to host immune suppression and compelling the need for designing non-immunosuppressive calcineurin inhibitors. In addition, due to the highly conserved nature of calcineurin molecule, it is important to obtain crystal structure of $A$. fumigatus calcineurin in order to design strategies to specifically target calcineurin in the fungal pathogen without affecting the host. Identification of the unique SPRR in filamentous fungal calcineurin is one such domain that can be pursued for potential targeting. Our finding of differential phosphorylation of calcineurin at the SPRR in the presence of micafungin and caspofungin indicates the probability of these antifungals inhibiting the phosphorylation of calcineurin by blocking the kinase responsible for phosphorylation. Identification of the kinase and inhibiting its activity can be another potential alternative. Additional strategies should involve characterization of the whole calcineurin interactome and phosphoproteome to define specific cell wall biosynthesis related interactors that are regulated by calcineurin through dephosphorylation. Data from such analyses may lead to the identification of potential new alternative targets and the design of better dual targeting strategies. For instance, our recent characterization of the two major heat shock proteins in A. fumigatus (Hsp90 and Hsp70) revealed their interaction with calcineurin [55] and a positive interaction between FK506 and geldanamycin (Hsp90 inhibitor), leading to fungicidal effect was noted [52,56,57].

\section{Conclusions}

As a critical phosphatase, calcineurin may regulate several downstream targets through dephosphorylation and contribute to the activation/deactivation of numerous effector proteins and possibly other signaling pathways. Calcineurin control over hyphal morphogenesis, septation, cell wall integrity, and stress adaptation, may all contribute to fungal virulence. Despite being a drug target, the immunosuppressive nature of the current calcineurin inhibitors is an obstacle to overcome, and designing non-immunosuppressive anti-calcineurin agents that are fungal-specific is challenging. Over the last decade, our studies have provided new insights into its role in hyphal growth and septation. Exploiting some of the unique aspects of fungal calcineurin, such as phosphorylation or identification of its interactions with fungal specific structures such as the cell wall and septum machinery regulators, may lead us to new targets to explore.

\section{Acknowledgments}

Research work presented in this review was supported by grants from the NIH/NIAID (R01 AI112595-01, R56AI077648-01A2, R21AI097541-01A1) to WJS. 


\section{Author Contributions}

Praveen R Juvvadi and William J Steinbach conceived and designed the research. Praveen R Juvvadi performed the experiments. Praveen R Juvvadi and William J Steinbach wrote the manuscript. Praveen R Juvvadi and William J Steinbach approved the final submission.

\section{Conflicts of Interest}

The authors declare no conflict of interest.

\section{References}

1. Kontoyiannis, D.P.; Marr, K.A.; Park, B.J.; Alexander, B.D.; Anaissie, E.J.; Walsh, T.J.; Ito, J.; Andes, D.R.; Baddley, J.W.; Brown, J.M.; et al. Prospective surveillance for invasive fungal infections in hematopoietic stem cell transplant recipients, 2001-2006: Overview of the transplant-associated infection surveillance network (transnet) database. Clin. Infect. Dis. 2010, 50, 1091-1100.

2. Hemenway, C.; Heitman, J. Calcineurin. Cell Biochem. Biophys. 1999, 30, 115-151.

3. Ho, S.; Clipstone, N.; Timmermann, L.; Northrop, J.; Graef, I.; Fiorentino, D.; Nourse, J.; Crabtree, G.R. The mechanism of action of cyclosporin A and fk506. Clin. Immunol. Immunopathol. 1996, 80, S40-S45.

4. Breuder, T.; Hemenway, C.S.; Movva, N.R.; Cardenas, M.E.; Heitman, J. Calcineurin is essential in cyclosporin a- and fk506-sensitive yeast strains. Proc. Natl. Acad. Sci. USA 1994, 91, 5372-5376.

5. Cardenas, M.E.; Muir, R.S.; Breuder, T.; Heitman, J. Targets of immunophilin-immunosuppressant complexes are distinct highly conserved regions of calcineurin A. EMBO J. 1995, 14, 2772-2783.

6. Klee, C.B.; Crouch, T.H.; Krinks, M.H. Calcineurin: A calcium- and calmodulin-binding protein of the nervous system. Proc. Natl. Acad. Sci. USA 1979, 76, 6270-6273.

7. Rusnak, F.; Mertz, P. Calcineurin: Form and function. Physiol. Rev. 2000, 80, 1483-1521.

8. Rumi-Masante, J.; Rusinga, F.I.; Lester, T.E.; Dunlap, T.B.; Williams, T.D.; Dunker, A.K.; Weis, D.D.; Creamer, T.P. Structural basis for activation of calcineurin by calmodulin. J. Mol. Biol. 2012, 415, 307-317.

9. King, M.M.; Huang, C.Y. The calmodulin-dependent activation and deactivation of the phosphoprotein phosphatase, calcineurin, and the effect of nucleotides, pyrophosphate, and divalent metal ions. Identification of calcineurin as a $\mathrm{Zn}$ and Fe metalloenzyme. J. Biol. Chem. 1984, 259, 8847-8856.

10. Juvvadi, P.R.; Lamoth, F.; Steinbach, W.J. Calcineurin as a multifunctional regulator: Unraveling novel functions in fungal stress responses, hyphal growth, drug resistance, and pathogenesis. Fungal Biol. Rev. 2014, 28, 56-69.

11. Cyert, M.S.; Kunisawa, R.; Kaim, D.; Thorner, J. Yeast has homologs (CNA1 and CNA2 gene products) of mammalian calcineurin, a calmodulin-regulated phosphoprotein phosphatase. Proc. Natl. Acad. Sci. USA 1991, 88, 7376-7380.

12. Cyert, M.S. Calcineurin signaling in saccharomyces cerevisiae: How yeast go crazy in response to stress. Biochem. Biophys. Res. Commun. 2003, 311, 1143-1150. 
13. Matsumoto, T.K.; Ellsmore, A.J.; Cessna, S.G.; Low, P.S.; Pardo, J.M.; Bressan, R.A.; Hasegawa, P.M. An osmotically induced cytosolic $\mathrm{Ca}^{2+}$ transient activates calcineurin signaling to mediate ion homeostasis and salt tolerance of Saccharomyces cerevisiae. J. Biol. Chem. 2002, 277, 33075-33080.

14. Mendoza, I.; Quintero, F.J.; Bressan, R.A.; Hasegawa, P.M.; Pardo, J.M. Activated calcineurin confers high tolerance to ion stress and alters the budding pattern and cell morphology of yeast cells. J. Biol. Chem. 1996, 271, 23061-23067.

15. Mendoza, I.; Rubio, F.; Rodriguez-Navarro, A.; Pardo, J.M. The protein phosphatase calcineurin is essential for nacl tolerance of Saccharomyces cerevisiae. J. Biol. Chem. 1994, 269, 8792-8796.

16. Sugiura, R.; Sio, S.O.; Shuntoh, H.; Kuno, T. Calcineurin phosphatase in signal transduction: Lessons from fission yeast. Genes Cells 2002, 7, 619-627.

17. Yoshida, T.; Toda, T.; Yanagida, M. A calcineurin-like gene ppb1+ in fission yeast: Mutant defects in cytokinesis, cell polarity, mating and spindle pole body positioning. J. Cell Sci. 1994, 107, 1725-1735.

18. Lu, Y.; Sugiura, R.; Yada, T.; Cheng, H.; Sio, S.O.; Shuntoh, H.; Kuno, T. Calcineurin is implicated in the regulation of the septation initiation network in fission yeast. Genes Cells 2002, 7, 1009-1019.

19. Kume, K.; Koyano, T.; Kanai, M.; Toda, T.; Hirata, D. Calcineurin ensures a link between the DNA replication checkpoint and microtubule-dependent polarized growth. Nat. Cell Biol. 2011, 13, 234-242.

20. Chen, Y.L.; Kozubowski, L.; Cardenas, M.E.; Heitman, J. On the roles of calcineurin in fungal growth and pathogenesis. Curr. Fungal Infect. Rep. 2010, 4, 244-255.

21. Kozubowski, L.; Heitman, J. Profiling a killer, the development of Cryptococcus neoformans. FEMS Microbiol. Rev. 2012, 36, 78-94.

22. Sanglard, D.; Ischer, F.; Marchetti, O.; Entenza, J.; Bille, J. Calcineurin a of Candida albicans: Involvement in antifungal tolerance, cell morphogenesis and virulence. Mol. Microbiol. 2003, 48, 959-976.

23. Cruz, M.C.; Fox, D.S.; Heitman, J. Calcineurin is required for hyphal elongation during mating and haploid fruiting in Cryptococcus neoformans. EMBO J. 2001, 20, 1020-1032.

24. Cruz, M.C.; Goldstein, A.L.; Blankenship, J.R.; del Poeta, M.; Davis, D.; Cardenas, M.E.; Perfect, J.R.; McCusker, J.H.; Heitman, J. Calcineurin is essential for survival during membrane stress in Candida albicans. EMBO J. 2002, 21, 546-559.

25. Kothe, G.O.; Free, S.J. Calcineurin subunit b is required for normal vegetative growth in Neurospora crassa. Fungal Genet. Biol. 1998, 23, 248-258.

26. Prokisch, H.; Yarden, O.; Dieminger, M.; Tropschug, M.; Barthelmess, I.B. Impairment of calcineurin function in Neurospora crassa reveals its essential role in hyphal growth, morphology and maintenance of the apical $\mathrm{Ca}^{2+}$ gradient. Mol. Gen. Genet. 1997, 256, 104-114.

27. Juvvadi, P.R.; Arioka, M.; Nakajima, H.; Kitamoto, K. Cloning and sequence analysis of cnaa gene encoding the catalytic subunit of calcineurin from Aspergillus oryzae. FEMS Microbiol. Lett. 2001, 204, 169-174.

28. Juvvadi, P.R.; Kuroki, Y.; Arioka, M.; Nakajima, H.; Kitamoto, K. Functional analysis of the calcineurin-encoding gene cnaa from Aspergillus oryzae: Evidence for its putative role in stress adaptation. Arch. Microbiol. 2003, 179, 416-422.

29. Nanthakumar, N.N.; Dayton, J.S.; Means, A.R. Role of $\mathrm{Ca}^{++} /$calmodulin binding proteins in Aspergillus nidulans cell cycle regulation. Prog. Cell Cycle Res. 1996, 2, 217-228. 
30. Harel, A.; Bercovich, S.; Yarden, O. Calcineurin is required for sclerotial development and pathogenicity of Sclerotinia sclerotiorum in an oxalic acid-independent manner. Mol. Plant Microbe Interact. 2006, 19, 682-693.

31. Choi, J.H.; Kim, Y.S.; Lee, Y.H. Functional analysis of mcna, a gene encoding a catalytic subunit of calcineurin, in the rice blast fungus Magnaporthe oryzae. J. Microbiol. Biotechnol. 2009, 19, 11-16.

32. Schumacher, J.; de Larrinoa, I.F.; Tudzynski, B. Calcineurin-responsive zinc finger transcription factor crz1 of Botrytis cinerea is required for growth, development, and full virulence on bean plants. Eukaryot. Cell 2008, 7, 584-601.

33. Egan, J.D.; García-Pedrajas, M.D.; Andrews, D.L.; Gold, S.E. Calcineurin is an antagonist to pka protein phosphorylation required for postmating filamentation and virulence, while pp2a is required for viability in Ustilago maydis. Mol. Plant Microbe Interact. 2009, 22, 1293-1301.

34. Cervantes-Chávez, J.A.; Ali, S.; Bakkeren, G. Response to environmental stresses, cell-wall integrity, and virulence are orchestrated through the calcineurin pathway in Ustilago hordei. Mol. Plant Microbe Interact. 2010, 24, 219-232.

35. Fox, D.S.; Heitman, J. Good fungi gone bad: The corruption of calcineurin. BioEssays 2002, 24, 894-903.

36. Yu, S.J.; Chang, Y.L.; Chen, Y.L. Calcineurin signaling: Lessons from candida species. FEMS Yeast Res. 2015, doi:10.1093/femsyr/fov016.

37. Steinbach, W.J.; Reedy, J.L.; Cramer, R.A.; Perfect, J.R.; Heitman, J. Harnessing calcineurin as a novel anti-infective agent against invasive fungal infections. Nat. Rev. Microbiol. 2007, 5, 418-430.

38. Lee, S.C.; Li, A.; Calo, S.; Heitman, J. Calcineurin plays key roles in the dimorphic transition and virulence of the human pathogenic zygomycete Mucor circinelloides. PLoS Pathog. 2013, 9, 289-290.

39. Lee, S.C.; Li, A.; Calo, S.; Inoue, M.; Tonthat, N.K.; Bain, J.M.; Louw, J.; Shinohara, M.L.; Erwig, L.P.; Schumacher, M.A.; et al. Calcineurin orchestrates dimorphic transitions, antifungal drug responses and host-pathogen interactions of the pathogenic mucoralean fungus Mucor circinelloides. Mol. Microbiol. 2015, 97, 844-865.

40. Steinbach, W.J.; Cramer, R.A.; Perfect, B.Z.; Asfaw, Y.G.; Sauer, T.C.; Najvar, L.K.; Kirkpatrick, W.R.; Patterson, T.F.; Benjamin, D.K., Jr.; Heitman, J.; et al. Calcineurin controls growth, morphology, and pathogenicity in Aspergillus fumigatus. Eukaryot. Cell 2006, 5, 1091-1103.

41. Da Silva Ferreira, M. E.; Heinekamp, T.; Härtl, A.; Brakhage, A.A.; Semighini, C.P.; Harris, S.D.; Savoldi, M.; de Gouvêa, P.F.; da Silva Goldman, M.H.; Goldman, G.H. Functional characterization of the Aspergillus fumigatus calcineurin. Fungal Genet. Biol. 2007, 44, 219-230.

42. Juvvadi, P.R.; Fortwendel, J.R.; Rogg, L.E.; Burns, K.A.; Randell, S.H.; Steinbach, W.J. Localization and activity of the calcineurin catalytic and regulatory subunit complex at the septum is essential for hyphal elongation and proper septation in Aspergillus fumigatus. Mol. Microbiol. 2011, 82, 1235-1259.

43. Juvvadi, P.R.; Fortwendel, J.R.; Pinchai, N.; Perfect, B.Z.; Heitman, J.; Steinbach, W.J. Calcineurin localizes to the hyphal septum in Aspergillus fumigatus: Implications for septum formation and conidiophore development. Eukaryot. Cell 2008, 7, 1606-1610. 
44. Ichinomiya, M.; Yamada, E.; Yamashita, S.; Ohta, A.; Horiuchi, H. Class i and class ii chitin synthases are involved in septum formation in the filamentous fungus Aspergillus nidulans. Eukaryot. Cell 2005, 4, 1125-1136.

45. Fukuda, K.; Yamada, K.; Deoka, K.; Yamashita, S.; Ohta, A.; Horiuchi, H. Class iii chitin synthase chsb of aspergillus nidulans localizes at the sites of polarized cell wall synthesis and is required for conidial development. Eukaryot. Cell 2009, 8, 945-956.

46. Muszkieta, L.; Aimanianda, V.; Mellado, E.; Gribaldo, S.; Alcàzar-Fuoli, L.; Szewczyk, E.; Prevost, M.C.; Latgé, J.P. Deciphering the role of the chitin synthase families 1 and 2 in the in vivo and in vitro growth of Aspergillus fumigatus by multiple gene targeting deletion. Cell. Microbiol. 2014, 16, 1784-1805.

47. Juvvadi, P.R.; Gehrke, C.; Fortwendel, J.R.; Lamoth, F.; Soderblom, E.J.; Cook, E.C.; Hast, M.A.; Asfaw, Y.G.; Moseley, M.A.; Creamer, T.P.; et al. Phosphorylation of calcineurin at a novel serine-proline rich region orchestrates hyphal growth and virulence in Aspergillus fumigatus. PLoS Pathog. 2013, 9, doi:10.1371/journal.ppat.1003564.

48. Falloon, K.; Juvvadi, P.R.; Richards, A.D.; Vargas-Muñiz, J.M.; Renshaw, H.; Steinbach, W.J. Characterization of the fkbp12-encoding genes in Aspergillus fumigatus. PLoS ONE 2015, 10, doi:10.1371/journal.pone.0137869.

49. Juvvadi, P.R.; Muñoz, A.; Lamoth, F.; Soderblom, E.J.; Moseley, M.A.; Read, N.D.; Steinbach, W.J. Calcium-mediated induction of paradoxical growth following caspofungin treatment is associated with calcineurin activation and phosphorylation in Aspergillus fumigatus. Antimicrob. Agents Chemother. 2015, 59, 4946-4955.

50. Steinbach, W.J. Are we there yet? Recent progress in the molecular diagnosis and novel antifungal targeting of Aspergillus fumigatus and invasive aspergillosis. PLoS Pathog. 2013, 9, doi:10.1371/journal.ppat.1003642.

51. Lamoth, F.; Juvvadi, P.R.; Steinbach, W.J. Histone deacetylase inhibition as an alternative strategy against invasive aspergillosis. Front. Microbiol. 2015, doi:10.3389/fmicb.2015.00096.

52. Lamoth, F.; Juvvadi, P.R.; Gehrke, C.; Steinbach, W.J. In vitro activity of calcineurin and heat shock protein 90 inhibitors against Aspergillus fumigatus azole- and echinocandin-resistant strains. Antimicrob. Agents Chemother. 2013, 57, 1035-1039.

53. Steinbach, W.J.; Schell, W.A.; Blankenship, J.R.; Onyewu, C.; Heitman, J.; Perfect, J.R. In vitro interactions between antifungals and immunosuppressants against Aspergillus fumigatus. Antimicrob. Agents Chemother. 2004, 48, 1664-1669.

54. Fortwendel, J.R.; Juvvadi, P.R.; Perfect, B.Z.; Rogg, L.E.; Perfect, J.R.; Steinbach, W.J. Transcriptional regulation of chitin synthases by calcineurin controls paradoxical growth of Aspergillus fumigatus in response to caspofungin. Antimicrob. Agents Chemother. 2010, 54, 15551563.

55. Lamoth, F.; Juvvadi, P.R.; Soderblom, E.J.; Moseley, M.A.; Steinbach, W.J. Hsp70 and the cochaperone stia (hop) orchestrate hsp90-mediated caspofungin tolerance in Aspergillus fumigatus. Antimicrob. Agents Chemother. 2015, 59, 4727-4733. 
56. Lamoth, F.; Juvvadi, P.R.; Fortwendel, J.R.; Steinbach, W.J. Heat shock protein 90 is required for conidiation and cell wall integrity in Aspergillus fumigatus. Eukaryot. Cell 2012, 11, 1324-1332.

57. Lamoth, F.; Alexander, B.D.; Juvvadi, P.R.; Steinbach, W.J. Antifungal activity of compounds targeting the hsp90-calcineurin pathway against various mould species. J. Antimicrob. Chemother. 2015, 70, 1408-1411.

(C) 2015 by the authors; licensee MDPI, Basel, Switzerland. This article is an open access article distributed under the terms and conditions of the Creative Commons Attribution license (http://creativecommons.org/licenses/by/4.0/). 